\title{
Special Issue ESIAM19 International Journal of Fatigue
}

Additive manufacturing offers the potential to economically fabricate customized parts with complex geometries in a rapid design-tomanufacture cycle. However, the basic understanding of the fatigue behaviour of these materials must be substantially improved at all scale levels before the unique features of this rapidly developing technology can be used in critical load bearing applications. This ambitious target can be reached solely via adventurous interdisciplinary research and our proposed project crosses materials science, technological processes, structural integrity, and advanced design methodologies.

As far as airframe and ground vehicle applications are concerned, developing a better understanding of fatigue performance is the key. Due to the novelty of this technology, a very limited literature exists on the fatigue performance of additively manufactured alloys. In particular, the physical phenomena linked to fatigue crack initiation and propagation in additively manufactured metals have not yet been investigated in detail, so that no standards and recommendation for fatigue design (both in the high- and in the low-fatigue regime) are available to date. Both theoretical understanding and structural applications are especially affected by this tremendous limitation. Hence, to achieve a better understanding of the basic fatigue phenomena and to provide industries with an effective fatigue assessment criterion will have a tremendous impact on several key industrial sector which include, amongst others, automotive, aerospace, and biomedical components/devises. For automotive industry, in particular, these advances in knowledge will create new opportunities in terms of innovative design, this resulting in lighter and safer products, shorter lead times, and lower production costs.

ESIAM 19 (The first European Conference on the Structural Integrity of Additively Manufactured Materials) has been the first conference organized by ESIS TC15 on the topics of fatigue and fracture of AM materials. The objective has been to provide an update state of the art on the development of effective criteria for the design of unprecedented high performing components for next generation automotive, aerospace and biomedical applications.

The conference was successfully held in Trondheim from $9^{\text {th }}$ to $11^{\text {th }}$ of September 2019 with more than 150 participants from more than 20 countries. The present special issue contains invited papers from the conference that were subjected to the standard peer review process usually performed in International Journal of Fatigue. The main focus of the special issue is fatigue design and assessment of additively manufactured materials under different loading scenarios.

As guest editors we want to express our acknowledgement to the authors, the reviewers and the editorial office staff that make this issue possible. We hope that the present issue provides a useful state of the art for engineers, academicians and industries involved in the challenges of producing high performant additively manufactured parts.

The next conference ESIAM 21 will be held in Vienna Austria from $8^{\text {th }}$ to $10^{\text {th }}$ September 2021 .

Sincerely yours

Filippo Berto Norwegian University of Science and Technology

Luca Susmel University of Shieffield

Brecht Van Hooreweder Ku Leuven

Jan Torgersen Norwegian University of Science and Technology

Guian Qian Institute of Mechanics, Chinese Academy of Sciences 